\title{
Synergistic combination of systems for structural health monitoring and earthquake early warning for structural health prognosis and diagnosis
}

\author{
Stephen $\mathrm{Wu}^{*^{\mathrm{a}}}$, James L. Beck*a \\ ${ }^{a}$ California Institute of Technology, 1200 E. California Blvd, Pasadena, CA, USA 91125
}

\begin{abstract}
Earthquake early warning (EEW) systems are currently operating nationwide in Japan and are in beta-testing in California. Such a system detects an earthquake initiation using online signals from a seismic sensor network and broadcasts a warning of the predicted location and magnitude a few seconds to a minute or so before an earthquake hits a site. Such a system can be used synergistically with installed structural health monitoring (SHM) systems to enhance pre-event prognosis and post-event diagnosis of structural health. For pre-event prognosis, the EEW system information can be used to make probabilistic predictions of the anticipated damage to a structure using seismic loss estimation methodologies from performance-based earthquake engineering. These predictions can support decision-making regarding the activation of appropriate mitigation systems, such as stopping traffic from entering a bridge that has a predicted high probability of damage. Since the time between warning and arrival of the strong shaking is very short, probabilistic predictions must be rapidly calculated and the decision making automated for the mitigation actions. For post-event diagnosis, the SHM sensor data can be used in Bayesian updating of the probabilistic damage predictions with the EEW predictions as a prior. Appropriate Bayesian methods for SHM have been published. In this paper, we use pre-trained surrogate models (or emulators) based on machine learning methods to make fast damage and loss predictions that are then used in a cost-benefit decision framework for activation of a mitigation measure. A simple illustrative example of an infrastructure application is presented.
\end{abstract}

Keywords: Earthquake Early Warning, Structural Health Monitoring, Bayesian Probability, Damage Prediction, Damage Assessment, Decision Support System, Prognosis and Diagnosis

\section{INTRODUCTION}

In the past decade, attention to structural health monitoring (SHM) has been significantly enhanced. Theory and hardware development for SHM has become one of the major subjects in structural engineering research. Most of the existing SHM methodologies rely on detecting a change in a specific structural property, the local change of stiffness, to evaluate potential damage in the structure and its severity. These methods collect response data from a deployed sensor network periodically or before and after an event, and estimate stiffness change based on a linear structural model. A common approach is to use Bayesian updating, or simply least-squares minimization, based on the difference between modal parameters calculated from a parameterized finite-element structural model and the corresponding modal frequency and mode shapes identified from a dynamic tes ${ }^{[23]}$, usually performed with low-amplitude ambient vibrations. The investigation may be performed for damage assessment after a severe excitation, such as earthquake loading, or for long-term monitoring of deterioration due to fatigue and corrosion.

Our interest in a robust SHM system is as a tool for post-earthquake structural damage and loss assessment. Acknowledging the development of earthquake early warning (EEW) systems in recent years, we believe that it would be valuable for a SHM system to be able to access information from EEW in order to improve its robustness in damage detection and loss estimation. In a practical SHM network, the number of response channels is usually much less than the degrees of freedom needed to accurately capture structural behavior. This condition of incomplete information motivates our use of a probabilistic approach in a SHM methodology. However, due to the level of complexity in this

\footnotetext{
*stewu@caltech.edu; jimbeck@caltech.edu
} 
problem, such as modeling uncertainty, measurement noise, etc., a large resulting uncertainty is present. The synergy with EEW offers an opportunity to reduce uncertainty based on the extra information.

In this paper, a framework for synergy between SHM and EEW is proposed for enhanced seismic protection of a target structure. The framework is divided into two parts: pre-event and post-event. For the pre-event case, EEW provides a few seconds to a minute or so of early warning before the destructive earthquake waves hit the target site. Therefore, a quick damage prediction can be made based on the warning information and used by a decision support system to decide whether to initiate an action to mitigate potential earthquake loss for the target structure ${ }^{[10,24]}$. The EEW information can also be exploited to assist the control of a structural sensor network immediately before an earthquake. Also, a graphical structural map of predicted structural damage or loss can be constructed and continuously updated using the available information. For the post-event case, fast damage detection can be conducted using a probability-based SHM approach, which combines the sensor information with EEW information to obtain robust damage assessment. The EEW damage or loss structural map can be updated for post-event assessment. In the following, we will first provide background information on a probabilistic SHM approach and on EEW development. Then, the details of a synergistic framework between SHW and EEW are illustrated. Lastly, we will conclude with a simple example and future work.

\section{BACKGROUND}

\subsection{Bayesian probabilistic approach to SHM data analysis}

The fundamental concept for model-based SHM is to update a structural model based on measured data to determine local loss of stiffness, which is taken to represent corresponding local structural damage in the actual structure. The simplest method is to minimize a measure of the difference between the identified modal parameters from response data and those calculated from a pre-determined structural model. This approach involves a modal identification procedure. A generic objective function to be minimized is then a weighted sum of squares of the difference between the identified modal frequencies and mode shapes and those determined from a finite element model ${ }^{[26]}$ :

$$
J=\sum_{i=1}^{m} \alpha_{i}\left(\omega_{i}-\hat{\omega}_{i}\right)^{2}+\sum_{i=1}^{m} \beta_{i}\left\|\phi_{i}-\hat{\phi}\right\|^{2}
$$

where $m$ is the total number of modes included; $\alpha$ and $\beta$ are the chosen weighting factors; $\omega$ and $\phi$ are the natural frequencies and mode shapes from the structural model, respectively; $\hat{\omega}$ and $\hat{\phi}$ are the natural frequencies and mode shapes identified from response data, respectively. A major challenge for this approach is so-called mode matching, which is to match the modes determined by the structural model with those determined by measured data. In practice, the limited amount of instrumentation that may not cover sufficient degrees of freedom to characterize higher modes, together with imperfect structural models, create a large uncertainty for mode matching. Furthermore, uncertainty about the predictive capability of any structural model, as well as the lack of sensitivity of the modal parameters to localized stiffness change, create additional challenges. Therefore, a single model is often not enough to provide reasonable results for SHM. Another difficulty is how to choose appropriate weights $\alpha$ and $\beta$ in (1) since their values can have a significant effect on the optimal estimates of the model parameters.

In order to account for these large uncertainties, a Bayesian model updating approach provides a robust framework for $\mathrm{SHM}^{[4,23]}$. In this Bayesian framework, instead of a single plausible model, a set of models is considered with a derived posterior probability density function (PDF) over the set indicating their relative plausibility based on the data. Let $\theta$ be the vector of model parameters. First, we establish the prior PDF of $\theta$ for a model class $M, p(\theta \mid M)$, based on engineering judgment and pre-damage structural analysis. Then, the PDF is updated to the posterior PDF, $p(\theta \mid D, M)$, based on measured data $D$ using Bayes' theorem:

$$
p(\theta \mid D, M)=c \cdot p(D \mid \theta, M) p(\theta \mid M)
$$

where $c$ is the normalizing constant for the posterior PDF and $p(D \mid \theta, M)$ is the probability of the measured data given the model parameters, also known as the likelihood function of $\theta$. There are two major challenges in this Bayesian approach: 1) The evaluation of the likelihood function may be computationally intensive; 2) The normalizing constant $c$, which involves integrating the product of the likelihood and the prior with respect to $\theta$, may also be hard to evaluate. 
Many improvements in the Bayesian framework for SHM have been developed in the past decade: the idea of "expanded mode shapes" is developed to avoid mode matching ${ }^{[3,6]}$; Gibbs MCMC sampling ${ }^{[7]}$ of the posterior PDF was used to improve on the Laplace asymptotic method ${ }^{[4]}$ employed in the original framework $^{[23]}$; the eigenvalue equation was used in the prior to avoid any eigenvalue analysis of the structural model ${ }^{[26]}$; a method directly using the dynamic timedomain data ${ }^{[27]}$ was introduced to avoid the modal identification step; and data-based selection of the weighing factors $\alpha$ and $\beta$ was performed using Bayesian model class assessment ${ }^{[11]}$.

\subsection{Development of EEW and its applications}

Despite a lot of research effort in seismology, accurate prediction of when an earthquake will happen is still not possible due to the high uncertainty of the stress and strength distributions within the Earth's tectonic plates. Nevertheless, the rapid development of computing power and network communication has made a new technology possible: Earthquake Early Warning (EEW). An earthquake excites various kinds of waves, including P-waves, S-waves and surface waves. The P-wave is the fastest traveling wave with the least destructive power, while $\mathrm{S}$-wave and the surface waves are slower waves with much larger destructive power. EEW exploits two important speed differences to provide early warning before the destructive waves arrive a site: 1) The speed difference between the P-waves and S-waves; 2) The speed difference between seismic waves and electronic signals ${ }^{[20]}$. Once a seismic network detects P-wave information, EEW will perform fast prediction of the earthquake magnitude, hypocenter location and origin time. As the seismic waves propagate, the seismic network will receive more data and continuously update the EEW predictions. Since the electronic signals travel a lot faster than seismic waves, the warning time provided by EEW system depends heavily on the distance between a site and the hypocenter. General warning time is around a few seconds to a minute or so.

Japan has had a national EEW system for roughly 6 years, and other regions, such as Taiwan, Istanbul in Turkey, Mexico City and Bucharest in Romania, have more localized regional systems ${ }^{[2]}$. Currently, an EEW system called the California Integrated Seismic Network (CISN) ShakeAlert System is also under beta-testing in California, USA. As currently planned, ShakeAlert is a relatively unique system because it will combine the outputs of three early warning algorithms, each based on a different theory: $\tau_{\mathrm{c}}-\mathrm{P}_{\mathrm{d}}$ on-site algorithm ${ }^{[5]}$, Earthquake Alarms Systems (ElarmS) ${ }^{[1]}$, and Virtual Seismologist (V-S) ${ }^{[9]}$ (see Fig.1). All three systems receive data from the same CISN seismic network and run their own algorithm to produce PDFs of earthquake magnitude, location estimation and origin time. Their output will be integrated in a central decision module, which will produce a PDF for earthquake magnitude and location.

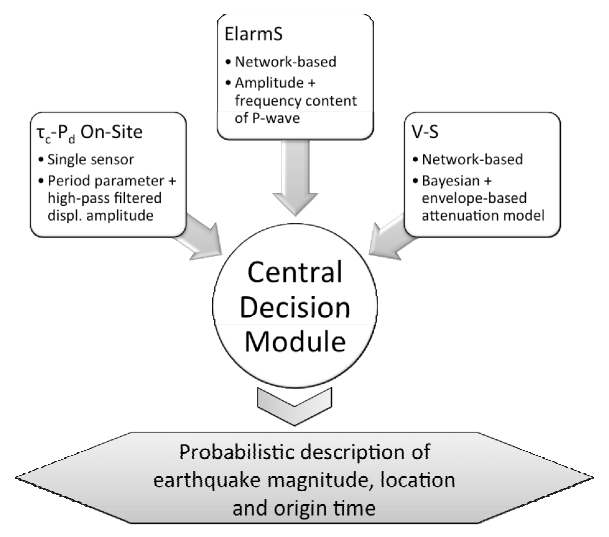

Figure 1. Planned structure of CISN ShakeAlert system

Despite the rapid development of EEW around the world, its engineering applications lag behind. Due to the uncertainty of EEW predictions and short warning times, existing applications are only those with a low activation cost and a simple procedure, such as elevator control ${ }^{[15]}$. One of the most successful engineering applications of EEW is the Urgent Earthquake Detection and Alarm System (UrEDAS) of the Japan Railway Group ${ }^{[17]}$ for the high-speed Shinkansen trains. Recently, the concept of integrating EEW with SHM in local damage detection ${ }^{[19]}$ or global loss assessment ${ }^{[14]}$ are mentioned by some research groups in Europe. In this paper, our aim is to development a complete synergistic framework between EEW and SHM to optimize the use of the information provided by both systems under a robust Bayesian probabilistic framework. 


\section{SYNERGY BETWEEN SHM AND EEW}

\subsection{Proposed synergistic framework}

EEW provides valuable earthquake information in the early stage of an earthquake event. SHM provides structural response data for post-event damage detection and loss assessment. Both systems can be combined into a single framework to provide a continuous decision-support system. Figure 2 shows a timeline comparing the original SHM framework with the synergistic framework.

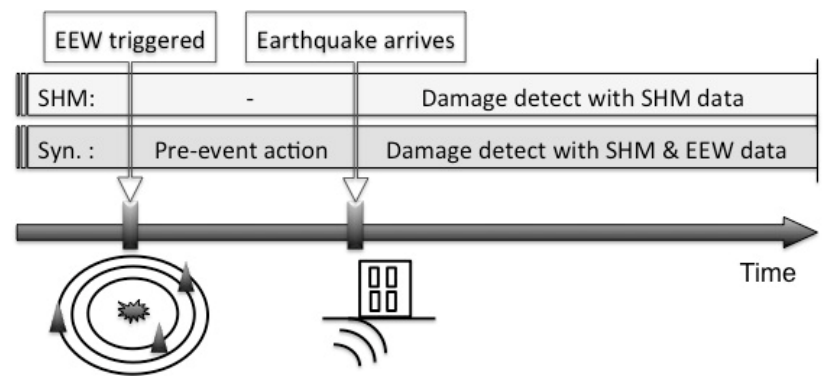

Figure 2. Timeline comparison between the SHM framework and the synergistic framework that combines information from EEW and SHM

A decision support system for damage assessment that only relies on post-earthquake structural response data suffers from significant uncertainty. The synergistic framework aims to exploit the EEW information to potentially reduce the uncertainty of the SHM damage prediction, and to allow loss mitigation with pre-event actions. This framework is composed of two stages. The first stage is the time between the EEW trigger and the earthquake arriving at a site. During this time, there are continual updates of the EEW prediction that will be fed into an automated decision support system for taking pre-event actions in order to mitigate the potential earthquake loss ${ }^{[24]}$. The automated decision is based on a cost-benefit analysis that takes the optimal action, including no action, in order to minimize expected loss. The costbenefit analysis is performed using the PEER performance-based earthquake engineering methodology (PBEE) ${ }^{[18]}$. A surrogate model can be constructed using an advanced machine learning technique, called the relevance vector machine $(\mathrm{RVM})^{[21,22]}$, in order to increase the efficiency of the real-time loss computations. The second stage starts after the earthquake arrives at the site of interest and structural response data becomes available from the SHM sensor network. By combining the information from EEW and SHM, more robust damage detection and assessment can be performed for a target structure. The details of a synergistic method based on EEW and SHM data are described in Section 3.3.

Table 1. Summary of decision support provided by the synergistic framework in the two stages.

\begin{tabular}{|l|l|}
\hline \multicolumn{1}{|c|}{ Stage } & \multicolumn{1}{|c|}{ Decision support } \\
\hline 1. After EEW triggered & $\begin{array}{l}\text { Mitigation action decision based on } \\
\text { cost-benefit analysis with EEW data }\end{array}$ \\
\hline 2. After earthquake arrives & $\begin{array}{l}\text { Damage detection with Bayesian } \\
\text { approach based on EEW \& SHM data }\end{array}$ \\
\hline
\end{tabular}

\subsection{Overview of the automated decision-making}

Two fundamental problems of EEW that restrict its applications are: short warning time and large uncertainty. If we know for sure that an earthquake is going to soon strike a structure and induce a response of sufficient severity, then planned mitigation actions can be taken immediately in order to mitigate potential earthquake losses. In reality, the early warning information will involve some uncertainty and some EEW applications will suffer a substantial economic loss if a false alarm occurs. Therefore, the decision of whether to take a mitigation action should involve sophisticated costbenefit analysis. However, human intervention during this process would likely use up too much of the short warning time, preventing the mitigation actions from being activated in a timely manner. This motivates our work to develop an 
earthquake probability-based automated decision-making (ePAD) system. Figure 3 shows a brief introduction of ePAD's fundamental concepts.

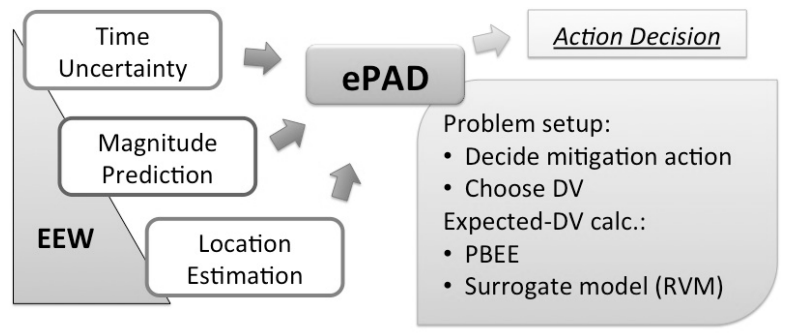

Figure 3. Basic structure of ePAD: DV stands for Decision Variable ${ }^{[10]}$

The cost-benefit analysis for EEW applications is highly dependent on the time remaining for actions, which is a key differentiator compared to other structural cost-benefit analyses. With continual updates of EEW information, ePAD involves a sequential decision problem, and we have proposed an automated decision making procedure that treats the limited time for action in a simplified manner in the cost-benefit analyses ${ }^{[24]}$. In an on-going research project, we are explicitly building the uncertainty in the remaining time until the arrival of strong shaking into the cost-benefit analyses.

\subsection{Methodology for synergy of EEW and SHM data}

The fundamental concept for synergy of EEW and SHM data is to take the EEW data as prior information to establish the prior PDF in (2) for the Bayesian SHM model. Since EEW provides extra information about the shaking expected at a site, hence the expected damage of a structure compared to our original knowledge about it, the EEW-based prior can potentially reduce the uncertainty in the SHM-based posterior PDF of damage in different substructures. A quantitative way of evaluating the benefits of the proposed synergy is introduced in the next section. A major challenge of this synergy method is the inconsistent metric for a local damage measure in existing EEW and SHM frameworks. One of the well-known earthquake loss assessment frameworks, known as the PEER PBEE methodology ${ }^{[12,18]}$, defines the damage state probability distributions for each damageable component using fragility curves, where the damage state variable, $D M$, is a set of discrete states. For example, $D M$ for a structural component would have possible values $\left\{D M_{1}\right.$, $\left.D M_{2}, \ldots, D M_{n}\right\}$ representing n possible damage states of the component and the fragility function gives the probability that the component is in state $D M_{i}$ given the demand on the component ${ }^{[12]}$. On the other hand, as shown in Section 2.1, the Bayesian probabilistic approach for SHM usually defines a local damage measure in terms of continuous variables, $\theta$, which is typically a vector of fractional stiffness loss in all substructures in a building. Therefore, the synergy between EEW and SHM requires a probabilistic relationship for $D M_{i}$ in EEW in terms of $\theta$ in SHM or vice versa, in order to consistently evaluate potential damage or loss after an earthquake.

This motivates two ways of evaluating the posterior PDF of $\left.D M, p\left(D M \mid D_{S}, D_{E}\right): 1\right)$ Updating from the prior PDF of $D M$, $p\left(D M \mid D_{E}\right)$ or 2) Updating from the prior PDF of $\theta, p\left(\theta \mid D_{E}\right)$, where the prior represents the damage state prediction, $D M$ or $\theta$, based on EEW data, $D_{E}$; while the posterior represents the damage state prediction updated with SHM data, $D_{S}$. In order to better illustrate the difference between the two proposed methods, we utilize the concept of Bayesian (Belief) Network (BN) to develop two models that represent the two methods, as shown in Figure 4.
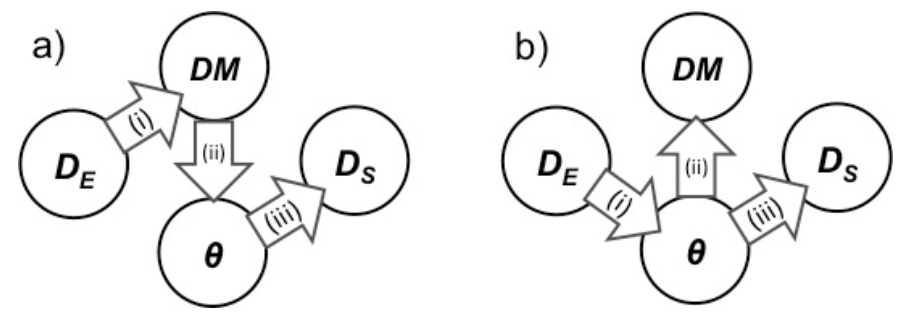

Figure 4. Bayesian network models. (a) In-series model; (b) $\theta$-centered model. (i) Prior PDF, $p\left(D M \mid D_{E}\right)$ or $p\left(\theta \mid D_{E}\right)$; (ii) Relationship between $D M$ and $\theta, p(D M \mid \theta)$ or $p(\theta \mid D M)$; (iii) Structural response model, $p\left(D_{S} \mid \theta\right)$ 


\section{Method 1: In-series BN model (define $\theta$ in terms of DM)}

By first using Bayes' theorem, and then expanding the likelihood function, $p\left(D_{S} \mid D M, D_{E}\right)$, with the Total Probability Theorem, one can calculate the posterior by:

$$
p\left(D M \mid D_{S}, D_{E}\right)=\frac{p\left(D_{S} \mid D M, D_{E}\right) p\left(D M \mid D_{E}\right)}{p\left(D_{S} \mid D_{E}\right)}=\frac{\int p\left(D_{S} \mid \theta, D M, D_{E}\right) p\left(\theta \mid D M, D_{E}\right) d \theta \cdot p\left(D M \mid D_{E}\right)}{p\left(D_{S} \mid D_{E}\right)}
$$

However, since (3) is still too complicated for practical use, for the "In-series BN model", we simplify some of the PDFs. Under this model, as shown in Figure 4(a), $D_{S}$ is independent (in the information sense) of both $D M$ and $D_{E}$ when $\theta$ is given, which means that $p\left(D_{S} \mid \theta, D M, D_{E}\right)$ equals $p\left(D_{S} \mid \theta\right)$. Also, $\theta$ is independent of $D_{E}$ when $D M$ is given, which means that $p\left(\theta \mid D M, D_{E}\right)$ equals $p(\theta \mid D M)$. Therefore, (3) can be simplified to:

$$
p\left(D M \mid D_{S}, D_{E}\right)=\frac{\int p\left(D_{S} \mid \theta\right) p(\theta \mid D M) d \theta \cdot p\left(D M \mid D_{E}\right)}{p\left(D_{S} \mid D_{E}\right)}
$$

Note that $p\left(D_{S} \mid \theta\right)$ in (4) corresponds to the likelihood function $p(D \mid \theta, M)$ in (2). Hence, any existing methodologies used for evaluating $p(D \mid \theta, M)$ in (2) can be used to evaluate $p\left(D_{S} \mid \theta\right)$ in (4). Also, the prior, $p\left(D M \mid D_{E}\right)$, is a known PDF calculated from the PBEE framework and $p\left(D_{S} \mid D_{E}\right)$ is simply a normalizing constant for the posterior PDF. The only unknown PDF left is $p(\theta \mid D M)$. This could be developed by engineering judgment and nonlinear deteriorating hysteretic structural analyses using a Gaussian model with a chosen mean and standard deviation that depend on DM. The advantage of this method is that there are no additional PDFs beyond those used in existing EEW and SHM frameworks except the required probabilistic relationship for $\theta$ in terms of $D M$. The disadvantage is that relating a set of continuous variables $\theta$ in terms of a set of discrete variables $D M$ may be relatively difficult, compared with the other way around.

\section{Method 2: $\theta$-centered $B N$ model (define DM in terms of $\theta$ )}

By first expanding the posterior of $D M$ with the Total Probability Theorem, and then applying Bayes' theorem to the posterior of $\theta, p\left(\theta \mid D_{S}, D_{E}\right)$, one can calculate the posterior of $D M$ by:

$$
p\left(D M \mid D_{S}, D_{E}\right)=\int p\left(D M \mid \theta, D_{S}, D_{E}\right) p\left(\theta \mid D_{S}, D_{E}\right) d \theta=\frac{\int p\left(D M \mid \theta, D_{S}, D_{E}\right) p\left(D_{S} \mid \theta, D_{E}\right) p\left(\theta \mid D_{E}\right) d \theta}{p\left(D_{S} \mid D_{E}\right)}
$$

Similar to Method 1, for the " $\theta$-centered BN model", we simplify some of the PDFs in (5). Under this model, as shown in Figure 4(b), $D M$ is independent of both $D_{E}$ and $D_{S}$ when $\theta$ is given, which means that $p\left(D M \mid \theta, D_{S}, D_{E}\right)$ equals $p(D M \mid \theta)$. Also, $D_{S}$ is independent of $D_{E}$ when $\theta$ is given, which means that $p\left(D_{S} \mid \theta, D_{E}\right)$ equals $p\left(D_{S} \mid \theta\right)$. Thus, (5) can be simplified to:

$$
p\left(D M \mid D_{S}, D_{E}\right)=\frac{\int p(D M \mid \theta) p\left(D_{S} \mid \theta\right) p\left(\theta \mid D_{E}\right) d \theta}{p\left(D_{S} \mid D_{E}\right)}
$$

Again, $p\left(D_{S} \mid \theta\right)$ is known from the SHM framework, $p\left(D_{S} \mid D_{E}\right)$ is a normalizing constant and $p(D M \mid \theta)$ is the established probabilistic relationship for $D M$ in terms of $\theta$ that must be established. The only other PDF to be established is the prior of $\theta, p\left(\theta \mid D_{E}\right)$, which can be calculated based on the first two steps of the four-step PBEE methodology:

$$
p\left(\theta \mid D_{E}\right)=\int p(\theta \mid I M) p\left(I M \mid D_{E}\right) d(I M)
$$

In (7), a surrogate model for $p(\theta \mid I M)$ in terms of the current substructure stiffness established based on synthetic training data using a nonlinear deteriorating hysteretic structural model. Also, $p\left(I M \mid D_{E}\right)$ can be established from the EEW data and a ground motion prediction equation for shaking intensity measure $I M$. The advantage of Method 2 is that definition of a set of discrete variables in terms of a set of continuous variables is relatively straightforward. For example, one can define each damage state $D M_{i}$ as a range of $\theta^{[23]}$, which means $p\left(D M_{i} \mid \theta\right)=1$ for the corresponding fixed range of $\theta$. Furthermore, in this model, since $D_{E}$ is directly fed into the SHM system, the EEW-based decision-support system and the modified SHM system can be run in parallel and combined at the end stage. A qualitative example is given later in Section 4 to illustrate the exact structure in practice. The disadvantage is that evaluation of $\mathrm{p}\left(\theta \mid D_{E}\right)$ is not often included in common PBEE loss assessments. Therefore, extra structural analyses are required before actual application. 
Note that by comparing (4) and (6), and using the product axiom of probability:

$$
p\left(D M, \theta \mid D_{E}\right)=p(D M \mid \theta) p\left(\theta \mid D_{E}\right)=p(\theta \mid D M) p\left(D M \mid D_{E}\right)
$$

it can be shown mathematically that the two methods are equivalent, as long as the probability relations of $\theta$ and $D M$ in terms of each other are consistent. Therefore, one may choose either of the two methods depending on the situation.

\subsection{Evaluation of the benefits of the proposed synergy}

A main benefit of the synergy framework based on Bayes' theorem is to reduce the uncertainty of the posterior PDF by introducing extra information from EEW data. Thus, this imposes an important question of how large the benefit is, or in other words, how much extra information do we actually gain from EEW data $D_{E}$. This question can be answered quantitatively using information theory. A direct comparison can be done using relative entropy (R.E. ${ }^{[8]}$, also known as Kullback-Leibler divergence, which is often used as a non-symmetric measure of the difference between two PDFs for the same variable, $\mathrm{X}$, but it is also a measure of the information gain in bits about $X$ from information $I$ :

$$
\text { R.E. }(X \mid I)=\int p(X \mid I) \cdot \log _{2}\left(\frac{p(X \mid I)}{p(X)}\right) d X
$$

We will use it as a measure of the information gain about $D M$ or $\theta$ from the EEW data $D_{E}$, by comparing the posterior with and without $D_{E}$. In Method $1, D_{E}$ is used to predict $D M$. Hence, we calculate R.E. $\left(D M \mid D_{E}\right)$ by setting $p(X \mid I)$ equal to $p\left(D M \mid D_{S}, D_{E}\right)$ and $p(X)$ equal to $p\left(D M \mid D_{S}\right)$ and then after some manipulation based on the Bayes' theorem:

$$
\text { R.E. }\left(D M \mid D_{E}\right)=\int p\left(D M \mid D_{S}, D_{E}\right) \cdot \log _{2}\left(\frac{p\left(D M \mid D_{E}\right)}{p(D M)}\right) d(D M)+\log _{2}\left(\frac{p\left(D_{S}\right)}{p\left(D_{S} \mid D_{E}\right)}\right)
$$

It is interesting to note that by adding a constant term that only depends on the data, R.E. $\left(D M \mid D_{E}\right)$ is influenced by the information difference between using the EEW prior and the original prior, which is exactly what we want to measure. In Method 2, since $D_{E}$ is used to predict $\theta$, we evaluate R.E. $\left(\theta \mid D_{E}\right)$, instead of R.E. $\left(D M \mid D_{E}\right)$. Again, the expression can be manipulated to give:

$$
\text { R.E. }\left(\theta \mid D_{E}\right)=\int p\left(\theta \mid D_{S}, D_{E}\right) \cdot \log _{2}\left(\frac{p\left(\theta \mid D_{E}\right)}{p(\theta)}\right) d(\theta)+\log _{2}\left(\frac{p\left(D_{S}\right)}{p\left(D_{S} \mid D_{E}\right)}\right)
$$

Note that in (10), we assume $p\left(D_{S} \mid D M, D_{E}\right)=p\left(D_{S} \mid D M\right)$ and in (11), we assume $p\left(D_{S} \mid \theta, D_{E}\right)=p\left(D_{S} \mid \theta\right)$ as implied by the $\mathrm{BN}$ models (a) and (b), respectively, in Figure 4.

\section{ILLUSTRATIVE EXAMPLE FOR SYNERGISTIC APPLICATION}

Consider an example based on a benchmark structure which is located on deep sediment near the center of the Los Angeles basin, at $33.996^{\circ} \mathrm{N}, 118.162 \circ \mathrm{W}$, south of downtown Los Angeles, and which is a four-story reinforced concrete moment-frame building designed according to the 2003 International Building Code. A detailed study, including its structural models, fragility curves and annual loss assessment, can be found in $[12,13]$. In this example, we illustrate how to exploit the synergistic framework using Method 2.

In the setup stage, we need to first define $D M$ and $\theta$, and construct the relationship between them. $D M$ is usually defined in such a way that it is convenient to perform loss assessment under the PEER PBEE framework. In this case, a set of damage states is already well-defined in $[12,13]$ for both structural and non-structural elements. Therefore, $D M$ for each structural component would have possible values $\left\{D M_{1}, D M_{2}, \ldots, D M_{n}\right\}$ representing $n$ possible damage states of the component and the fragility function gives the probability that the component is in state $D M_{i}$ given the demand on the component. $\theta$ is usually related to local stiffness loss in a structure. In this case, we divide the structure into 4 substructures, which each represents a single story of the structure, then $\theta=\left[\theta_{1}, \theta_{2}, \theta_{3}, \theta_{4}\right]$, where $\theta_{i}$ for $i=1 \ldots 4$ represents the fraction of the nominal substructure contribution, $K_{i}$, to the global stiffness matrix, $K(\theta)$, which is calculated from a finite-element model for the undamaged structure ${ }^{[23]}$ : 


$$
K(\theta)=K_{0}+\sum_{i=1}^{4} \theta_{i} K_{i}
$$

In Method 2, we need a relationship for $D M$ in terms of $\theta$. First, let us define $D M_{S}$ to be the damage state of a structural component, and $D M_{N S}$ to be the damage state of a non-structural component. For each such $D M_{S}$, a straightforward relation is to define a critical stiffness loss fraction, $d_{S i}$, for the stiffness parameter $\theta_{i}$ :

$$
P\left(D M_{S} \mid \theta_{i}\right)=P\left(\theta_{i} \leq\left(1-d_{S i}\right)\right)
$$

For each $D M_{N S}$, if the non-structural component's damage state is highly correlated to a substructure's stiffness loss, then (13) can be used. However, it is likely that some non-structural components' damage state cannot be clearly observed based on structural stiffness loss. In this case, choosing not to update the damage state PDF with $D_{S}$ may be a better choice, because inaccurate relations may lead to inaccurate predictions. Second, $p(\theta \mid I M)$ are needed to calculate the priors, $p\left(\theta \mid D_{E}\right)$, respectively, based on the PEER PBEE methodology $y^{[7,18]}$. The former PDF is simply the fragility curves for each damage state. The latter can be obtained in a similar fashion using the structural models that are already developed for constructing fragility curves. Since $\theta_{i}$ is a continuous variable, one may obtain a 3-D surface, instead of a 2-D curve, for $p\left(\theta_{i} \mid I M\right)$. Finally, a methodology for calculating the likelihood, $p\left(D_{S} \mid \theta\right)$, is needed. One may choose from the existing methods $[3,4,6,7,23,25,26,27]$.

Once the setup stage of the synergistic framework is finished, the system is ready to provide decision support and early loss prediction before an earthquake arrives, and also to provide updated loss assessment and safety alert after the earthquake arrives, as shown in Figure 5.

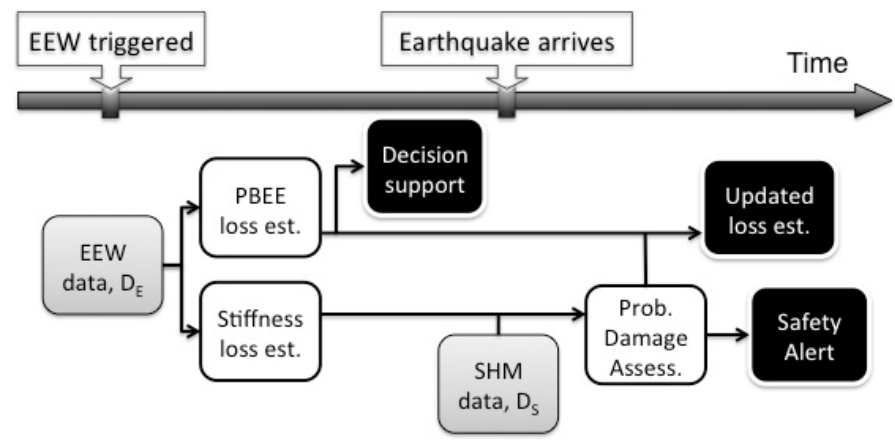

Figure 5. Flow chart of Method 2 application. The grey boxes represent input modules; the white boxes represent analysis modules; the black boxes represent output modules.

Note that the information from EEW is fed into the PBEE loss assessment system and SHM system independently under this method. Then, the SHM information is used to obtain the posterior PDF of $\theta, p\left(\theta \mid D_{S}, D_{E}\right)$, which is fed back to the PBEE system in order to update the loss estimation. For safety alert, one may directly use the posterior PDF of $\theta$, $p\left(\theta \mid D_{S}, D_{E}\right)$, obtained from the synergistic system to classify the damage level of a structure. However, a more practical approach is to use the feedback structure shown in Figure 5 to obtain the posterior PDF of $D M, p\left(D M \mid D_{S}, D_{E}\right)$, which can be used to perform virtual structural inspections using the Virtual Inspector ${ }^{[16]}$.

\section{CONCLUSION}

EEW can provide valuable information for civil structure applications. In this paper, we proposed a synergistic framework to utilize information from both EEW and SHM systems to provide more reliable loss estimation and safety alerts. EEW information can be used independently to provide pre-event loss estimation and decision support based on the PEER PBEE methodology. The original probabilistic SHM system provides damage assessment, but usually suffers from high uncertainty in the final prediction. Our approach is to use the EEW data as prior information to potentially reduce the prediction uncertainty in the SHM system. Also, the posterior information from the SHM system can be used as feedback information to update the EEW loss estimation and provide more reliable safety alerts after the earthquake is over by using the Virtual Inspector ${ }^{[16]}$. An evaluation method is also proposed to assess the potential benefit of using the synergistic system versus the original systems. A qualitative example has been used to demonstrate how this synergy 
works in practice. Our on-going work is to complete some quantitative results in order to verify the potential benefit of this approach.

\section{REFERENCES}

[1] Allen, R.M et al., "Real-time earthquake detection and hazard assessment by ElarmS across California," Geophysical Research Letters, 36(L00B08) (2009).

[2] Allen, R.M et al., "The status of earthquake early warning around the world: An introductory overview," Seismological Research Letters 80(5), 682-693 (2009).

[3] Beck, J. L., Au, S.K. and Vanik, M.W., "Monitoring structural health using a probabilistic measure," ComputerAided Civil and Infrastructure Engineering 16, 1-11 (2001).

[4] Beck, J. L. and Katafygiotis, L. S., "Updating models and their uncertainties. I: Bayesian statistical framework," Journal of Engineering Mechanics (ASCE) 124(4), 455-461 (1998).

[5] Bose, M. et al., "A new trigger criterion for improved real-time performance of onsite earthquake early warning in Southern California," Bulletin of the Seismological Society of America 99(2A), 897-905 (2009).

[6] Ching, J. and Beck, J.L., "Bayesian analysis of the phase II IASC-ASCE structural health monitoring experimental benchmark data," Journal of Engineering Mechanics (ASCE) 130(10), 1233-1244 (2004).

[7] Ching, J., Muto, M. and Beck, J. L., "Structural model updating and health monitoring with incomplete modal data using Gibbs sampler," Computer-Aided Civil and Infrastructure Engineering 21(4), 242-257 (2006).

[8] Cover, T. M. and Thomas, J. A., [Elements of Information Theory], Wiley-Interscience (2006).

[9] Cua, G. and Heaton, T., "The Virtual Seismologist (VS) method: a Bayesian approach to earthquake early warning," Earthquake Early Warning Systems, 97-132 (2007).

[10] Grasso, V. F., Beck, J. L. and Manfredi, G., "Automated decision procedure for earthquake early warning," Engineering Structures 29(12), 3455-3463 (2007).

[11] Goller, B., Beck, J. L. and Schueller, G. I., "Evidence-based identification of weighting factors in Bayesian model updating using modal data," Journal of Engineering Mechanics 138(5) (2012) [In Press].

[12] Goulet, C. A. et al., "Evaluation of the seismic performance of a code-conforming reinforced-concrete frame building-from seismic hazard to collapse safety and economic losses," Earthquake Engineering and Structural Dynamics 36(13), 1973-1997 (2007).

[13] Haselton, C. B. et al., "An assessment to benchmark the seismic performance of a code-conforming reinforced concrete moment-frame building," Pacific Earthquake Engineering Research Center Technical Report (2008).

[14] Hilbring, D. et al., "Earthquake early warning for transport lines," Natural Hazards (2010).

[15]Kubo, T. et al., "Applications of an earthquake early warning system and a real-time strong motion monitoring system in emergency response in a high-rise building," Soil Dynamics and Earthquake Engineering 31(2), 231-239 (2011).

[16] Mitrani-Reiser, J. and Beck, J. L., "Incorporating losses due to repair costs, downtime and fatalities in performancebased earthquake engineering," Proc. Computational Methods in Structural Dynamics and Earthquake Engineering (2007).

[17] Nakamura, Y., Sita, J. and Sato T., "On an earthquake early warning system (EEW) and its applications," Soil Dynamics and Earthquake Engineering 31(2), 127-136 (2011).

[18] Porter, K. A., “An overview of PEER's Performance-Based Earthquake Engineering methodology,” Applications of Statistics and Probability in Civil Engineering 1-2, 973-980 (2003).

[19] Rainieri, C., Fabbrocino, G. and Cosenza, E., "Integrated seismic early warning and structural health monitoring of critical civil infrastructures in seismically prone areas," Structural Health Monitoring - An International Journal 10(3), 291-308 (2011).

[20] Satriano, C., Wu, Y. M., Zollo, A. and Kanamori, H., "Earthquake early warning: Concepts, methods and physical grounds," Soil Dynamics and Earthquake Engineering 31(2), 106-118 (2011).

[21] Tipping, M. E., "Sparse Bayesian learning and the Relevance Vector Machine," Journal of Machine Learning Research 1(3), 211-244 (2001).

[22] Tipping, M. E. and Faul, A. C., "Fast marginal likelihood maximization for sparse Bayesian models," Proc. $9^{\text {th }}$ International Workshop on Artificial Intelligence and Statistics (2003). 
[23] Vanik, M. W., Beck, J. L. and Au, S. K., "Bayesian probabilistic approach to structural health monitoring," Journal of Engineering Mechanics (ASCE) 126(7), 738-745 (2000).

[24] Wu, S. and Beck, J.L., "An automated decision-making system framework for earthquake early warning system applications," Proc. 8CUEE Conference, 1737-1740 (2011).

[25] Yuen, K. V., Au, S. K. and Beck, J. L., "Two-stage structural health monitoring approach for phase I benchmark studies," Journal of Engineering Mechanics (ASCE) 130(1), 16-33 (2004).

[26] Yuen, K. V., Beck, J. L. and Katafygiotis, L. S., "Efficient model updating and health monitoring methodology using incomplete modal data without mode matching," Structural Control and Health Monitoring 13(1), 91-107 (2006).

[27] Yuen, K. V., Beck, J. L. and Katafygiotis, L. S., "Unified probabilistic approach for model updating and damage detection," Journal of Applied Mechanics (Transactions of the ASME) 73(4), 555-564 (2006). 